\title{
Airway Management in Pregnant Women With COVID-19
}

\author{
Amir Ghabousian' ${ }^{1}$, Ata Mahmoodpoor ${ }^{2 * \mathbb{D}}$
}

$\mathrm{T}$ The coronavirus disease 2019 (COVID-19) pandemic has represented a major impact to health systems and societies worldwide (1). Pregnant women do not appear to be more susceptible to infection or to serious complications compared to non-pregnant women (2). Presence of comorbidities may increase the risk for presenting with more severe clinical manifestations. Inflammation and host immune response lead to cytokine storm which results in organ dysfunction in COVID-19 with the lung as the most important organ and acute respiratory distress syndrome (ARDS) as the most severe form of complication resulting in intubation (3). Pregnant women are more prone to difficult airway management because of the physiologic changes (4). COVID-19 transmission is person to person by respiratory droplets after contact with an infected person. As these patients may need operations during their pregnancy, management of airway can be important and sometimes problematic in some cases $(5,6)$. Since the COVID-19 pandemic, faceto-face consultations have not been recommended and many interactions are now virtual. This has the potential to interact for accurate planning for airway assessment, in particular of pregnant women.

Every anesthesiologist should consider that the general anaesthesia is a high-risk procedure and therefore is not recommended as the first option for pregnant patients with COVID-19. General anaesthesia is recommended if the COVID-19 patient has $\mathrm{SpO} 2<93 \%$ (despite supplemental oxygen). Isolation of pregnant woman with COVID-19 is highly recommended in a negative pressure room. Before the operation, anaesthesiologist should consider preparing the devices and the necessary medication, planning the procedure, providing the proper personalised protective equipment, and double gloving. Because of possible difficult airway, alternative difficult tracheal intubation techniques consisted of tracheal intubation via a supraglottic airway or with video guidance and an Aintree intubation catheter may be needed and should be considered. A check list for airway/difficult airway

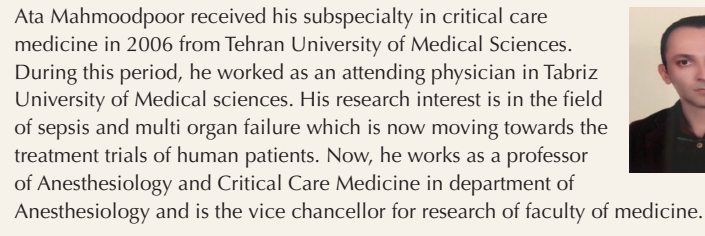

management may be suitable for this intervention and clear role allocation is necessary before starting. Intubation should be performed by the most experienced clinician $(7,8)$. For minimizing aerosol generating reaction like coughing, intravenous or topical lidocaine administration is recommended before initiation of intubation. Because of physiologic changes during pregnancy and COVID-19 infection, the obstetric patient is more susceptible to hypoxia, so pre-oxygenation is a critical step. A closed circuit is optimal (e.g. anaesthetic circle breathing circuit) and a rebreathing circuit (e.g. Mapleson's $\mathrm{C}$ circuit) is preferable to a bag-mask which expels virus containing exhaled gas into the room. Standard protocols are consisted of pre-oxygenation (some recommend high flow nasal canula) and rapid sequence induction without mask ventilation and the use of a second-generation video laryngoscope is recommended as an initial option which should be performed with a stylet with hyperangulated blade. Moreover, if using a bougie or stylet, caution must be taken when removing it so as not to spray secretions on the intubating team. Video laryngoscope allows the anaesthesiologist to maintain a greater distance from the patients' airway compared to direct laryngoscopy. Patient positioning, including ramping in the obese and reverse Trendelenburg positioning, should be adopted to maximize safe apnoea time. The number of people in the operating room during airway management should be minimized. After intubation, confirmation of tracheal tube should be performed by continuous waveform capnography and mechanical ventilation should only be administered after cuff inflation and ensuring no leakage. 
Using auscultation to confirm endotracheal tube position should be avoided. As extubation is an aerosol generating procedure, all precaution should be performed during it and especially peri-extubation coughing should prevented using dexmedetomidine, lidocaine or opioids $(9,10)$. Extubated patients should wear a surgical mask and if they need supplemental oxygen, the surgical mask should be placed over the nasal canula or $\mathrm{O} 2$ face mask. Also minimizing the number of staff during extubation in the operating room is necessary.

It should be reminded that multidisciplinary teamwork is fundamental in these patients, since the safety of many people is at stake and neuraxial anaesthesia and analgesia techniques appear to be safe in the obstetric patients with COVID-19 who are candidate for cesarean section. The airway management of pregnant patients with COVID-19 should be consisted of the safety for patient and healthcare workers; accuracy regarding avoiding unnecessary, unreliable and unfamiliar techniques; besides timely interventions without rush or delay. Increasing the knowledge and confidence level of healthcare workers regarding airway management of pregnant women with COVID-19 seems obligatory. We need further high quality researches to provide an evidence-based foundation for the airway management of pregnant patients with COVID-19. Physicians should work to decrease the impact of COVID-19 on maternal and fetal outcomes despite the loosing existed standards in other sectors of healthcare.

\section{Ethical Issues}

Not applicable.

\section{Conflict of Interests \\ None.}

\section{Authors' contributions}

AG: literature review, manuscript revising; AM: concept of study, initial drafting, manuscript revising. Both authors read and approved final draft of manuscript.

\section{References}

1. Mahmoodpoor A, Shadvar K, Ghamari AA, et al. Management of Critically III Patients with COVID-19: What We Learned and What We Do. Anesth Pain Med. 2020;10(3):e104900. Published 2020 Jun 10. doi:10.5812/aapm.104900

2. López M, Gonce A, Meler E, et al. Coronavirus Disease 2019 in Pregnancy: A Clinical Management Protocol and Considerations for Practice. Fetal Diagn Ther. 2020;47(7):519528. doi:10.1159/000508487

3. Hamishehkar $\mathrm{H}$, Beigmohammadi MT, Abdollahi $\mathrm{M}$, et al. Identification of enhanced cytokine generation following sepsis. Dream of magic bullet for mortality prediction and therapeutic evaluation. Daru. 2010;18(3):155-162.

4. Kurdoglu M, Khaki A. What is the current state of knowledge about the novel coronavirus infection during pregnancy? Int J Womens Health Reprod Sci. 2020;8(2):110-111. doi:10.15296/ijwhr.2020.17

5. Mahmoodpoor A. Role for the second anesthesiologist in failed intubations. Anesth Analg. 2006;102(3):971. doi: 10.1213/01. ANE.0000190879.76048.2D

6. Nader ND, Mahmoodpoor A. COVID-Associated Respiratory distress syndrome (CARDS) in pregnancy: What makes it so different? Int J Womens Health Reprod Sci. 2020;8(4):336337. doi:10.15296/ijwhr.2020.54.

7. Hernandez Gonzalez L. Anestesia general en la cesárea. Recomendaciones actuales. Actualización de los protocolos asistenciales de la Sección de Anestesia Obstétrica de la SEDAR. 2016:109-118.

8. The Faculty of Intensive Care Medicine. COVID-19 Airway management guidance. https://icmanaesthesiacovid-19.org/ airway-management-guidance. 2020.

9. Tung A, Fergusson NA, Ng N, Hu V, Dormuth C, Griesdale DEG. Medications to reduce emergence coughing after general anaesthesia with tracheal intubation: a systematic review and network meta-analysis. Br J Anaesth. 2020;S00070912(20)30012-X. doi:10.1016/j.bja.2019.12.041

10. Mahmoodpoor A, Ekrami E, Soleimanpour $\mathrm{H}$. Dexmedetomidine: An all sedation-in-one drug in critically ill patients with COVID-19. Pharm Sci 2020;26(suppl 1): S80-S81.

(C) 2021 The Author(s); This is an open-access article distributed under the terms of the Creative Commons Attribution License (http:// creativecommons.org/licenses/by/4.0), which permits unrestricted use, distribution, and reproduction in any medium, provided the original work is properly cited. 